\title{
A Spectrum Etiquette Protocol for Efficient Coordination of Radio Devices in Unlicensed Bands
}

\author{
D. Raychaudhuri and Xiangpeng Jing \\ WINLAB, Rutgers University \\ 73 Brett Road \\ Piscataway, NJ 08854, USA \\ Email: $\{$ ray, xjing\}@winlab.rutgers.edu
}

\begin{abstract}
This paper presents a spectrum etiquette protocol for efficient coordination of radio communication devices in unlicensed (e.g. 2.4 GHz ISM and 5 GHz U-NII) frequency bands. The proposed etiquette method enables spectrum coordination between multiple wireless devices using different radio technologies such as IEEE 802.11.x, 802.15.x, Bluetooth, Hiperlan, etc. The basic idea is to standardize a simple common protocol for announcement of radio and service parameters, called the "common spectrum coordination channel (CSCC)". The CSCC mechanism is based on the low bit-rate mode of the $802.11 b$ physical layer, along with a periodic broadcast protocol at the MAC layer. The CSCC protocol is "policy neutral" in the sense that it provides a general mechanism which can accommodate a wide range of specific spectrum sharing rules. One possible CSCC protocol implementation is described in terms of the packet formats used and related channel access rules. Proof-of-concept experimental results from a CSCC prototype are presented for an example scenario in which nearby $802.11 b$ and Bluetooth devices contend for $2.4 \mathrm{GHz}$ ISM band access. Results showing file transfer delay with and without CSCC etiquette are given for comparison purposes.
\end{abstract}

\section{INTRODUCTION}

Unlicensed frequency bands (such as the $2.4 \mathrm{GHz}$ ISM band and the $5 \mathrm{GHz}$ U-NII band) have played a critical role in enlarging the scope and penetration of wireless technology. The IEEE 802.11b wireless local-area network is the most notable example of proliferating unlicensed band wireless technologies for computer applications. As the popularity of unlicensed radio devices such as $802.11 \mathrm{~b}$ and Bluetooth grows, there is increasing concern about the potential for destructive interference between uncoordinated devices, particularly those with different radio access standards. There are increasing reports of problems in coordinating frequency and power settings of $802.11 \mathrm{~b}$ devices owned by different organizations or individuals, and of destructive interference between Bluetooth and $802.11 \mathrm{~b}$ devices [1]. These problems have motivated a renewed interest in spectrum etiquette for reducing destructive interference and improving overall spectrum utilization in unlicensed bands. The goal is to avoid the classic "tragedy of the commons" effect where the collective value of a shared resource (in this case, spectrum) is diminished by "overgrazing" due to the lack of cooperative procedures that balance individual needs with overall social utility.

The U.S. FCC in its 1998 U-NII ruling [2] indicated a preference for "technology neutral" spectrum etiquette policy that would permit co-existence and competition of multiple radio technologies, which may each be optimized for different applications. The technology neutral approach also facilitates rapid introduction of emerging radio technologies without the delays associated with traditional standards processes. Recent experience with wireless local-area and personal-area networks has shown that multiple standards are likely to co-exist at any given time, and that the recent "Moore's law" type evolution of radio technologies makes it unlikely that a single radio standard, however popular, will remain unchanged for more than five years (e.g. evolution of $802.11 \mathrm{~b}, \mathrm{a}, \mathrm{g}, . .$. ). All this argues for a renewed industry effort to standardize a flexible spectrum etiquette policy [3,4] that would work well with a variety of existing and emerging radio technologies intended for WLAN and WPAN scenarios. In this paper, we propose a specific spectrum etiquette mechanism called the "common spectrum coordination channel" or the "CSCC" [5, 6]. The concept is to enable mutual observability between neighboring radio devices via a simple common protocol by which each wireless device announces its radio parameters. It is noted that mutual observation is the foundation for all forms of "civil society" in which members can coordinate their behavior to meet their individual needs while paying attention to the "common good". Spectrum sharing has much in common with the usage of other public resources (such as parks, public spaces, waterways, etc.), and it is interesting to note that only the radio scenario is currently characterized by a complete lack of mutual awareness of users with potentially competing needs. The problem cannot be solved adequately by the previous generation of spectrum etiquette policies such as listen-before-talk (LBT) due to increasingly complex service requirements (e.g., media streaming with assured qualityof-service, low-delay communications, emergency response, etc.). Also, LBT can result in relatively low overall spectrum efficiency due to interference between signals that overlap partially in frequency or time and the lack of guidance towards selecting a "clear" channel or time-slot. This leads us to conclude that there is a real need for a more advanced type of etiquette protocol that provides a foundation for efficient radio resource sharing without the need for a single PHY/MAC standard in each band. Upon some reflection, it is clear that although improved LBT-type mechanisms could have limited value, a more general solution is going to require 
a common coordination channel typically implemented as a simple protocol operating at the edge of each unlicensed band. This approach does incur the additional cost of a dual-mode radio, but it can be argued that a modest increment in device cost (say $\sim \$ 5$ at current prices) is well worth the increase in end-user value (reliable services, QoS assurances, graceful degradation under congestion conditions) and societal value (improved utilization of public spectrum). Methods for reducing interference between different radio devices in unlicensed band have recently generated increasing interest. Most of the work in this area is on the coordination between WLAN and WPAN devices, in particular, between IEEE 802.11b and Bluetooth [7-9]. A lot of experimental and simulation work has been done on evaluating the interference between IEEE 802.11 and Bluetooth [9-11]. IEEE 802.15 WPAN Task Group 2 and Bluetooth SIG Coexistence WG are both developing recommended practices to facilitate coexistence of between 802.11 WLAN, and 802.15 or Bluetooth WPAN. For non-collaborative co-existence solutions for 802.11 and Bluetooth, individual devices detect the presence of other kinds of devices by measuring the bit or frame error rate, the signal strength, or the SNR (by RSSI). For example, Adaptive Frequency Hopping (AFH) [12] classifies channels and alters the regular hopping sequence to avoid channels with the most interference, and it is implemented in Bluetooth devices. AFH needs modifications of FCC rules, and hardwarelevel change of the hopping mechanism of Bluetooth radios. Collaborative algorithms [13] have also been proposed as an alternative method for coordination. In this work, we propose a spectrum etiquette protocol intended to support coordination between unlicensed band radio devices in a technology neutral manner. The details of a proposed CSCC etiquette protocol are discussed in the following sections. In section II, we introduce the system concept of the spectrum etiquette. In section III, we discuss the details of the protocol, including the protocol stack, CSCC packet format, CSCC MAC access and etiquette policies. Then some preliminary experimental results are shown as a proof-of-concept in section IV. Finally, we conclude the paper with comments on future work in section $\mathrm{V}$.

\section{SySTEM CONCEPT}

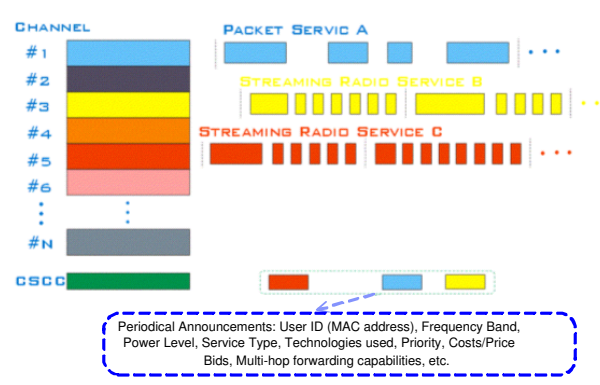

Fig. 1. Basic principle of CSCC etiquette protocol

The basic concept of the "common spectrum coordination channel (CSCC)" is illustrated in Fig. 1. The CSCC is a narrow control channel shared by all users of the band intended for spectrum coordination purposes. Each device has an extra narrow-band (low bit-rate) radio for exchange of control information over the CSCC channel. When different devices need to use spectrum, the CSCC method requires all users to periodically broadcast spectrum usage information (including: user ID such as IEEE MAC address, frequency band used and transmit power as well as optional parameters such as technology type, service type, multi-hop forwarding capabilities if any, user priority, etc.) using a simple standardized packet transmission protocol in the pre-defined sub-channel at the edge of the unlicensed band. Observation of these announcements permits newly active users to obtain a map of spectrum activity and select available frequencies, if any. All the CSCC broadcast is in an on-demand manner, which means only those devices that have spectrum request or those that are already transmitting will announce their spectrum usage information via the CSCC broadcast. Other users will remain silent and listen to the CSCC information. In the event that no clear channel is available when a device has a new spectrum request, it will transmit a contention message on the coordination channel. This initiates distributed execution of a specified etiquette procedure which results in distributed sharing of radio resources (i.e., frequency, power, time) in the congested region.

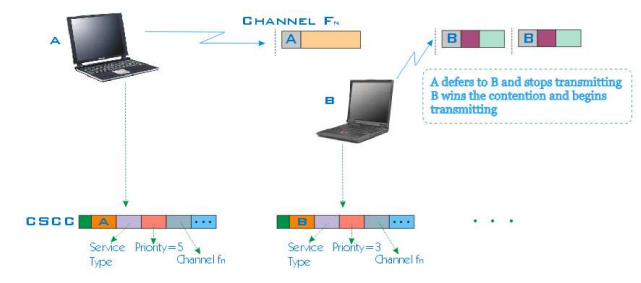

Fig. 2. Example of CSCC protocol used to execute a priority etiquette policy

An example in which the CSCC method is used to implement a simple priority-based etiquette policy is shown in Fig. 2. In this example, user $\mathrm{A}$ is already using the channel Fn. When user B has a spectrum request with higher priority than user A, it first listens for CSCC messages, which are broadcast periodically by all active devices within radio range. If no clear channel is available, B may decide to compete with user A for usage of channel Fn. Then user B announces itself by broadcasting a CSCC message in the control channel, informing others its service type, priority, preferred channel number and other information. When $\mathrm{A}$ receives this control message from its CSCC radio, it will defer to B and stop transmitting since it has a lower priority then user B. After this process, user $\mathrm{B}$ wins the contention and begins transmitting. The same mechanism may be used to implement a broad range of etiquette policies, including dynamic congestion pricing [14] in which contending users place actual price bids for usage of the channel. The advantage of the CSCC method is that it permits considerable flexibility in spectrum sharing procedures, which can now take into account more complex factors such as type of service or user priority consistent 
with public policy objectives. More advanced collaborative power control and/or multi-hop routing procedures may also be implemented within this type of framework. In addition, this method provides users with a "program guide" type capability as they enter a new area, thus simplifying terminal start-up procedures for access to network services.

\section{CSCC PROTOCOL}

In this section, we will discuss some implementation issues for the CSCC protocol, including the protocol description, protocol stack, packet format and timing parameters, etc. Various etiquette policies will also be discussed based on different criteria such as priority and pricing.

\section{A. CSCC Protocol Stack}

Fig. 3 shows the proposed dual-mode spectrum etiquette and data protocol stacks to be implemented by compliant unlicensed band radios. The spectrum etiquette protocol consists of standardized CSCC-PHY and CSCC-MAC layers with an etiquette policy module above. The spectrum etiquette (SE) policy module(s) must also be standardized for specific usage settings (e.g. home, indoor office, outdoor public, etc.) or for different regions, but these standards (including semantics for the parameters involved) can be set independently from the basic CSCC protocol.

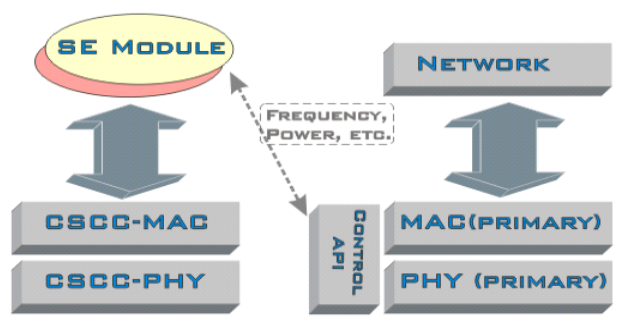

Fig. 3. CSCC protocol stack

For the CSCC-PHY, we can use the basic 1Mbps 802.11 standard, in order to leverage existing hardware designs, and to keep the complexity to a minimum. The $1 \mathrm{Mbps}$ mode of 802.11 at nominal $10 \mathrm{~mW}$ transmit power may be expected to provide $\sim 50-100 \mathrm{~m}$ coverage in most indoor and outdoor scenarios, sufficient for coordination in most unlicensed WLAN and WPAN scenarios. Lower powered WPAN devices with shorter range may reduce the transmit power on the CSCCPHY to correspond to a small multiple of their nominal radio range. The CSCC PHY must be standardized for edge-ofband operation in each unlicensed band, although it is also possible to consider a single band at the edge of either 2.4 or $5 \mathrm{GHz}$ unlicensed spectrum with control information at the MAC layer to cover multiple frequency bands.

\begin{tabular}{|c|c|c|c|c|c|}
\hline $\begin{array}{l}\text { Dest } \\
\text { addr }\end{array}$ & $\begin{array}{l}\text { Src } \\
\text { addr }\end{array}$ & Type & $\begin{array}{l}\text { IE } \\
\text { length }\end{array}$ & $\operatorname{IE}(1)$ & $\mathrm{IE}(\mathrm{n})$ \\
\hline $6 \mathrm{~B}$ & $6 \mathrm{~B}$ & $2 \mathrm{~B}$ & $2 \mathrm{~B}$ & $2 \mathrm{~B}$ & $2 \mathrm{~B}$ \\
\hline
\end{tabular}

Fig. 4. CSCC packet format (in bytes)
1) CSCC Packet Format: A possible CSCC-MAC layer packet format is shown in Fig. 4. A standard Ethernet packet format with control payload extensions is adopted as the basis. The 48-bit MAC address (source address) is used as the unique identifier, along with spectrum etiquette information elements for frequency band, power, etc. The semantics of these information elements is related to specific network conditions, frequency assignment, power control and (potentially) multihop collaboration algorithms to be used by a specific SE policy module. The Ethernet destination address is used to denote multicast groups that specify classes of potential neighbors which are expected to participate in the etiquette procedure. Also, the 2-byte type field can be used to indicate the specific SE policy to be used in connection with the information elements received over the CSCC.

For example, in the case of a scenario which IEEE 802.11b devices coexist with Bluetooth devices in a dense network, we can define several useful information elements: IE(1): Clamed Channel, IE(2): Priority, IE(3): Pricing based on bid, IE(4): Session Duration, IE(5): Transmitted Power Level, IE(6): Received Signal Strength. Other IE fields to support power control or more complex frequency/time coordination can also be defined as needed.

\section{B. CSCC MAC Access}

The CSCC MAC protocol itself is a simple periodic announcement protocol with randomization of the transmit cycle to eliminate repeated collisions. Each station transmits the CSCC packet periodically with a repetition interval of about $100 \mathrm{~ms}$ to a few seconds. The exact values depend on desired start-up and system response times.

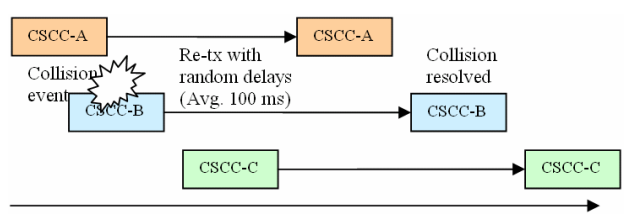

Fig. 5. CSCC MAC access with randomized periodic transmissions

1) CSCC Packet Format: The channel access procedure is outlined in Fig 5. The CSCC of user A collides with that of user $\mathrm{B}$, but this collision is resolved in future transmissions via randomization of the transmission interval. Note that this type of one-way broadcast MAC procedure is extremely simple, and requires very little logic for implementation.

\section{Etiquette Policies}

As mentioned earlier, various etiquette policies based on sharing the shared use of channel/time/frequency/power, user priorities or even micro-auctions can be considered. Priority is often used as a simple policy for coexistence between different classes of users, e.g. police/fire/ambulance and generalpurpose data users. Another interesting policy is based on dynamic pricing [14] based on micro-auctions between contending users. When the channel is congested, each user offers to pay a price for accessing spectrum resources, and the winner 
of the auction then proceeds to transmit. Efficient use of radio resources via agile radios and/or collaborative multi-hop routing models can also be implemented in this framework since the CSCC provides a map of current usage, eliminating the need for complex and slow frequency scanning procedures. A more advanced use of the CSCC is for "collaborative spectrum usage" in which multiple devices cluster together into a collaborative group that forms an ad-hoc network with multi-hop routing and power control. The CSCC can be used to advertise multi-hop routing capabilities and the willingness to join such a collaborative ad-hoc network of this type.

\section{PROOF-OF-CONCEPT PROTOTYPE}

In this section, we present some preliminary experimental results for CSCC applied to a simple network with both IEEE 802.11b and Bluetooth devices. The experiments are aimed at evaluating how the concept of our etiquette system works in a realistic environment with uncoordinated devices which potentially interfere with each other. The goals of the experiment are also to validate the protocol's operation, to evaluate protocol design options, and to study alternative spectrum sharing policies. The network scenario is very simple corresponding to two pairs of incompatible radio devices coexisting in a public space. As Fig. 6 shows, we have two pairs of radio devices, one 802.11b WLAN and one Bluetooth, in which Bluetooth1 and WLAN1 are senders and Bluetooth2 and WLAN2 are receivers.

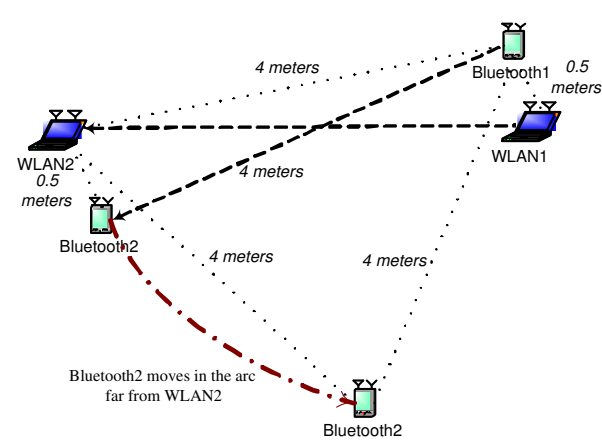

Fig. 6. Experimental network senario for devices with dual mode radio

Each of the radio devices is hosted on a laptop computer running Linux. All the devices are equipped with dual mode radios running $\mathrm{CSCC}$, using an $802.11 \mathrm{~b}$ radio at $1 \mathrm{Mbps}$ tuned at a different channel from the WLAN user card. Initially Bluetooth2 is near the WLAN2, and then it is moved away from WLAN2 while keeping the same distance from Bluetooth1. In our experiments, we evaluate the benefit of a priority-based etiquette protocol based for TCP file transfer services. The experimental parameters are listed in Table 1. The spectrum etiquette protocol itself is implemented in user space, and in this case consists of a simply priority-based on/off mechanism. The CSCC packet has six information elements as discussed in section III, encapsulated in the standard Ethernet packet in order to reuse existing WLAN drivers. Figs. 7 and 8 show comparative throughput traces vs. time for WLAN and Bluetooth (BT) data sessions with and without CSCC etiquette. When CSCC is turned on, WLAN and Bluetooth devices resolve contentions by using the priority etiquette and the winner continues to transmit without further interference.

TABLE I

EXPERIMENTAL PARAMETERS

\begin{tabular}{|c|c|c|}
\hline Mobility & WLAN nodes & Bluetooth nodes \\
\hline Traffic Model & 100M bytes data by TCP & $\begin{array}{c}\text { BT1 static, } \\
\text { BT2 position varies }\end{array}$ \\
\hline MAC protocol & $\begin{array}{c}1.5 \mathrm{M} \text { bytes data by } \\
\text { Stop-and-wait scheme }\end{array}$ \\
\hline Data Card & $\begin{array}{c}\text { Cisco Aironet } 350 \text { series } \\
\text { (at channel \#1) }\end{array}$ & $\begin{array}{c}\text { Bluetooth ACL data link } \\
\text { (hopping over whole band) }\end{array}$ \\
\hline CSCC MAC & IEEE 802.11 and periodic announcements at 1Mbps \\
\hline CSCC card & Cisco Aironet 350 series DS (at channel \#11) \\
\hline
\end{tabular}

It is observed that when WLAN users win, their throughputs can improve $\sim 35 \%$, and if BT users win, the throughput improvement is $\sim 30 \%$. It is also observed that without CSCC, BT devices cause periodic interference to WLAN, thus tending to close and open the TCP window repeatedly. The figures confirm that this degradation can be avoided by using the proposed etiquette protocol.

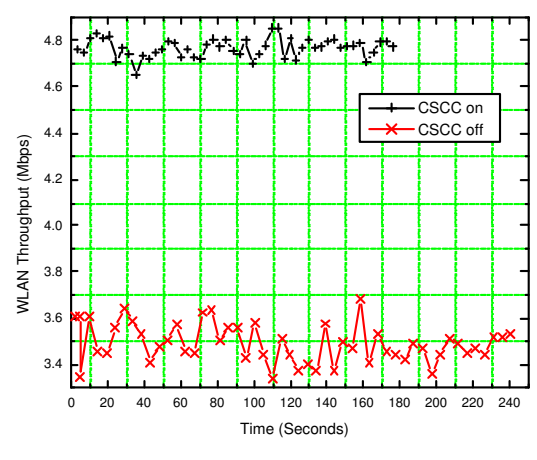

Fig. 7. Throughput trace for WLAN session with BT2 in initial position

To evaluate the total data session delay with and without CSCC etiquette, BT2 was moved away from WLAN2 as outlined in Fig. 6. WLAN session delays are reduced 12 30\% depending on distance, and BT session delays are reduced $15 \sim 22 \%$ as shown in Fig. 9. It is interesting to observe that as we move BT2 far from WLAN2 in an arc (while keeping the distance between BT1 and BT2 constant), the session delays for both WLAN and Bluetooth first decrease and then increase without CSCC. This is because BT2 is moving further from WLAN2 but nearer to WLAN1. So their interference first decreases and then increases. When CSCC is turned on, the session delay is almost constant since the two kinds of devices obtain spectrum resources in turn and there is no interference. Fig. 10 shows the instantaneous packet delay trace for a BT data session. When CSCC is turned on at 230 seconds, the 


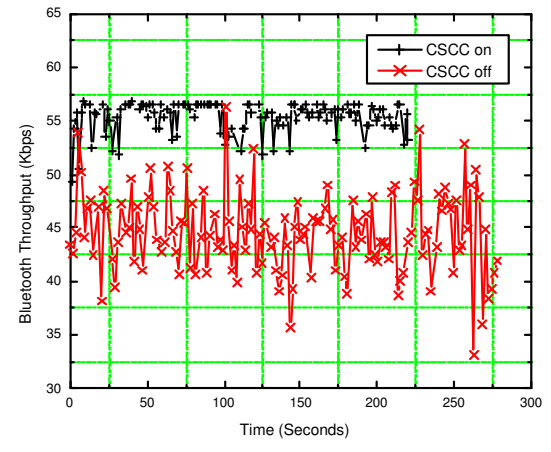

Fig. 8. Throughput trace for BT session with BT2 in initial position

BT user wins the contention with WLAN and its packet delay becomes lower and more stable than without CSCC.

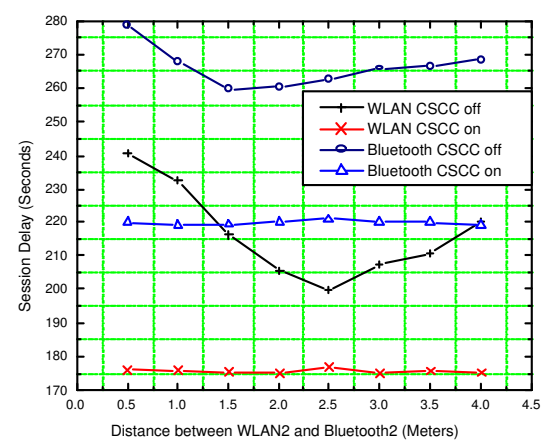

Fig. 9. Average session delay with and w/o CSCC vs. distance parameter

\section{CONCLUSiOn AND Future Work}

In this paper, we have proposed a spectrum etiquette protocol to coordinate radio devices in unlicensed bands. The proposed CSCC etiquette protocol provides a simple way for radio devices with different technologies to announce their own parameters in using a common coordination channel the edge of the unlicensed spectrum band. The CSCC message is periodically broadcast during the data session of the users so that resources such as frequency, power and time can be allocated in a fair and spectrally efficient manner. Different etiquette policies are also discussed depending on different network service conditions. Experimental results show that in the $2.4 \mathrm{GHz}$ ISM band, contending 802.11 and Bluetooth devices can achieve improved throughput and delay for both devices using simple priority-based etiquettes. Our current work is still in an early stage, focusing on the system concepts and evaluation of various protocol design options and etiquette algorithms. In future work, we plan to evaluate various levels of spectrum coordination with and without a formal etiquette protocol using both simulation and experiments. The options

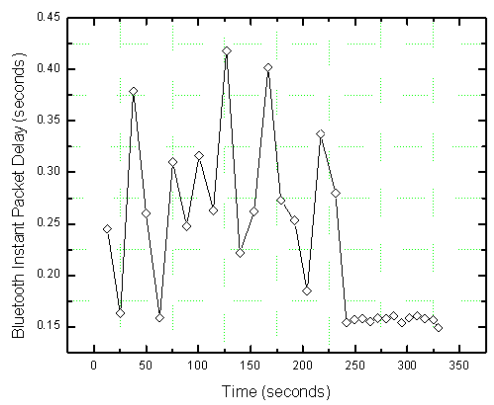

Fig. 10. Instantaneous packet delay for BT with CSCC turned on at $\mathrm{t}=230 \mathrm{~s}$

to be considered range from the simplest reactive source rate control scheme to priority and pricing based schemes (such as those discussed here) to more complex collaborative spectrum sharing methods involving ad-hoc networking and power control. The results will be made available to future spectrum policy and standardization processes concerned with efficient use of unlicensed spectrum.

\section{REFERENCES}

[1] D. P. Satapathy and J. M. Peha, "Spectrum Sharing Without Licenses: Opportunities and Dangers," in "Interconnection and the Internet," book of Selected Papers From The 1996 Telecommunications Policy Research Conference, G. Rosston and D. Waterman (Eds.), Mahwah, NJ.

[2] U.S. Federal Communications Communication, "Operation of Unlicensed NII Devices in the 5 GHz Range," ET Docket 96-102, Jan. 1997.

[3] D. P. Satapathy and J. M. Peha, "Performance of Unlicensed Devices With a Spectrum Etiquette," in Proceedings of IEEE Globecom, Nov. 1997.

[4] Durga P. Satapathy and Jon M. Peha, "Etiquette Modification for Unlicensed Spectrum: Approach and Impact," in Proceedings of the IEEE Vehicular Technology Conference, vol. 1, May 1998.

[5] D. Raychaudhuri, "Considerations for FCC's $5 \mathrm{GHz}$ NII/Supernet NPRM," ATM Forum/96-8058/WATM, June 1996.

[6] D. Raychaudhuri, "Wireless ATM and the U-NII Spectrum," Winlab FOCUS' 98 Workshop on the Unlicensed National Information Infrastructure, June 22-23, Long Beach, NJ.

[7] A Kamerman, "Coexistence between Bluetooth and IEEE 802.11 CCK Solutions to Avoid Mutual Interference," presentation to Lucent Technologies Bell Laboratories, Murray Hill, NJ, Jan. 1999.

[8] Marko Hnnikinen, Tapio Rantanen and Jani Ruotsalainen, et al., "Coexistence of Bluetooth and Wireless LANs," in the 8th edition of IEEE ICT, 2001.

[9] G. Ennis, "Impact of Bluetooth on 802.11 Direct Sequence," in IEEE P802.11 Working Group Contribution, IEEE P802.11-98/319, Sept. 1998.

[10] C.F. Chiasserini, R. Rao, "Performance of IEEE 802.11 WLANs in a Bluetooth Environment," in IEEE Wireless Communications and Networking Conference, WCNC 2000, Chicago, IL, Sept. 2000.

[11] N. Glomie, R. E. Van Dyck, A. Soltanian, "Interference of Bluetooth and IEEE 802.11: Simulation Modeling and Performance Evaluation," in Proceedings of the Fourth ACM International Workshop on Modeling, Analysis and Simulation of Wireless and Mobile Systems, MSWIM'01, Rome, Italy, July 2001.

[12] B. Treister, A. Batra, K.C. Chen, O. Eliezer, "Adapative Frequency Hopping: A Non-Collaborative Coexistence Mechanism," in IEEE P802.15 Working Group Contribution, IEEE P802.15-01/252r0, Orlando, FL, May 2001.

[13] J. Lansford, R. Nevo, E. Zehavi, "MEHTA: A Method for coexistence between co-located 802.11b and Bluetooth Systems," in IEEE P802.15 Working Group Contribution, IEEE P802.15-00/360r0, Nov. 2000.

[14] Cengiz Evci, Bernard Fino, "Spectrum Management, Pricing, and Efficiency Control in Broad-Band Wireless Communications," in Proceedings of the IEEE, vol. 89, No. 1, Jan. 2001. 